\title{
A Classic Clinical Case: Neutrophilic Eccrine Hidradenitis
}

\author{
Ana-Maria Copaescu ${ }^{a}$ Jean-François Castilloux ${ }^{b}$ \\ Myrna Chababi-Atallah ${ }^{c}$ Christian Sinave $^{d} \quad$ Janie Bertrand ${ }^{a}$ \\ Divisions of ${ }^{a}$ Dermatology, and ${ }^{b}$ Hematology and Oncology, Department of Medicine, \\ Université de Sherbrooke, 'Department of Pathology, and ${ }^{\mathrm{d}}$ Division of Infectiology, \\ Department of Microbiology and Infectiology, Centre Hospitalier Universitaire de \\ Sherbrooke, Sherbrooke, Qué., Canada
}

\section{Key Words}

Neutrophilic dermatosis · Neutrophilic eccrine hidradenitis · Facial cellulitis · Chemotherapy

\begin{abstract}
Background: Neutrophilic eccrine hidradenitis (NEH) is a rare condition described mostly in adult patients receiving chemotherapy for acute myelogenous leukemia. When it affects the facial region, it can mimic cellulitis and delay the diagnostic, thus proper recognition is essential. Objective: This article describes a classic case of NEH. We will review the diagnostic, the differential diagnostic (mostly cellulitis) and the management of this condition. Methods: After a literature review, the patient's file was properly studied in order to portray a clear picture of this condition. Medical photographs and appropriate physical examination upon presentation are also included. Results: The diagnostic for NEH was suggested by the clinical presentation and confirmed histopathologically (skin biopsy). Conclusion: The diagnostic of NEH is essential in order to prevent multiple unnecessary antibiotics.
\end{abstract}

(c) 2013 S. Karger AG, Basel

\section{Background}

The term was used for the first time in 1982 to describe the skin reaction in a patient undergoing chemotherapy for myeloblastic leukemia; neutrophilic eccrine hidradenitis (NEH) unfortunately remains a poorly understood concept. The diagnosis is made histologically and usually refers to a neutrophilic infiltration around the eccrine sweat glands and mostly around the proximal portion coils. 
NEH is usually described in relation to other neutrophilic dermatoses, a group of skin diseases characterized by an infiltration of the skin by normal polymorphonuclear neutrophils No infectious or other causes are identified [1]; thus, its clinical aspect makes NEH sometimes difficult to distinguish from the Sweet Syndrome, an entity that will not be addressed in detail in this article.

NEH is a rare condition, though epidemiology was not precisely studied for this condition. Neutrophilic dermatoses in granulocytopenic patients receiving chemotherapy occur at an incidence rate of $2.6 \%$ (according to an article published in 1995) [2]. Furthermore, some case reports describe a facial NEH that can mimic cellulitis. For these patients, proper recognition of NEH can prevent unnecessary usage of antibiotics.

In this article, we describe a classic case of NEH with facial involvement in a patient receiving chemotherapy for acute myelogenous leukemia. A literature review will also be discussed.

\section{Case Report}

A 58-year-old patient was admitted to the Centre Hospitalier Universitaire de Sherbrooke for weight loss, fatigue, sleep alteration, mouth pain and difficulty in mastication due to gingival infiltration. Except for chronic back pain and a 40-pack-year smoking history, the patient did not report any medical problems. His mother was known to suffer from leukemia. After proper investigations, he was diagnosed with acute myelogenous leukemia (AML-5b monoblastic and monocytic) and prescribed the $7+3$ induction chemotherapy protocol (table 1). This treatment combines cytarabine (days 1-7) with idarubicin (days 13) [3]. The patient also received amikacin (aminoglycoside) and meropenem for a febrile neutropenia and valacyclovir for orolabial herpes.

Twelve days after the beginning of his treatment, the patient was referred to the dermatology division because of an acute, non-itchy, non-scaly skin eruption that progressed over 2-3 days and affected various regions of his body (the face and neck region, arms, upper back, and legs).

The examination revealed dark erythematous, violaceous and edematous plaques on the right and left periorbital areas, on the nose root (fig. 1), and on the neck. The right forearm had a unique, dry, non-scaly, round and well-delimited purple plaque. Similar lesions were also detected on the right upper back (fig. 2) and legs. Some plaques seemed indurated. Several petechiae were also found, mostly on the thighs and ankles.

Scalp, nails, mucosa and genitalia were not involved. The rest of the physical exam was negative.

A 3-mm punch biopsy was done in the right upper lateral chest wall. Histological features consisted of an inflammatory neutrophilic infiltrate surrounding the deep part of the eccrine glands and edema of the dermis (fig. 3, fig. 4). These findings were consistent with a diagnosis of NEH. No leukocytoblastic vasculitis was observed. The periodic acid shiff coloration was negative for infection. Sweet syndrome was eliminated after this biopsy. Another skin specimen was sent for culture and was negative for bacteria, mycobacteria, mycoses and virus.

Following the confirmed diagnosis, symptomatic treatment with potent corticosteroid cream was enforced. The neutrophil count normalized without filgrastim after 3 weeks. As for the NEH, it slowly resolved with topical corticosteroid treatment, leaving faint, postinflammatory erythematous plaques in the areas described above. A preventive treatment 
with oral dapsone was recommended for a possible consolidation chemotherapy step involving cytarabine.

\section{Discussion}

NEH is a benign, neutrophilic dermatosis of unknown etiology. It is associated with an adult population diagnosed with leukemia and receiving chemotherapy. A computerized literature review by Bachmeyer and Aractingi [4] analyzed 51 patients from 1982 to 1998. From 1998 until May 2013, 34 other cases were published (Medline EBSCO). Several articles are mentioned below.

While considering this diagnosis, one must first rule out an infection. Tissue cultures for bacteria, mycobacteria, fungi and viruses must be considered and are generally negative.

In this case, the patient presented erythematous and edematous plaques in the periorbital region, mimicking orbital cellulitis. Other cases have been reported in the literature. Six patients receiving induction chemotherapy with cytarabine for acute myelogenous leukemia presented facial plaques with similar descriptions [5-8]. However, observations regarding the evolution of the plaques are poor except for 3 of the cases in whom the lesions slowly extended across the face [5, 7] or neck-trunk region [7]. Finally, all of the 6 patients presented with febrile neutropenia.

Interestingly, we found one article describing a patient with periorbital, violaceous patches to acetaminophen [9]. Ultimately, facial NEH was also described in 1 healthy patient with no medical history [10].

Is it clinically possible to distinguish periorbital NEH from facial cellulitis? One characteristic is the color. In NEH, the lesions are dark, erythematous, violaceous, or purpuric compared to cellulitis, where erythema with poorly defined margins (or sharp borders in erysipelas) is described. Secondly, cellulitis involves pain and tenderness. Clinical cases have described NEH with tender and painful lesions [11]. Also, warmth of the affected area was not used to describe NEH, but may contribute to a cellulitis diagnosis. Finally, edema is found in both conditions. Cellulitis continues to be a far more common clinical problem and the final NEH diagnosis remains histopathological.

Nevertheless, case reports inform us that infectious causes for NEH do exist. Infectious agents such as HIV [12-14], Gram-positive cocci [15], Streptoccoccus spp. (endocarditis [16]), Serratia marescens [17], Nocardia spp. [18], Enterobacter cloacae [4] and Staphyloccocus aureus [4] were reported in patients who presented classical histological features of NEH.

As mentioned above, the diagnosis is histological and the biopsy demonstrates a neutrophilic infiltrate of the eccrine unit, edema of the dermis, and necrosis of the eccrine coils and glands. The mechanism by which the eccrine sweat unit is affected remains unknown. One hypothesis underlines the drug-induced direct cytotoxic effect. Toxic byproducts are secreted through sweat, and thus, by neutrophilic chemotaxis induced by cellular damage, infiltration with neutrophils of the eccrine coils and glands occurs [4]. Consequently, the toxic concentration of the drugs within the eccrine gland may lead to necrosis of the epithelial cells [8]. Even so, no studies that evaluated the drug concentration in sweat glands were found.

Another model describes NEH as a part of the neutrophilic dermatoses spectrum [1], as a paraneoplastic condition. As mentioned, NEH has been described in a healthy population as well [10]. Therefore, a theory regarding possible sweat gland abnormalities was formulated [4]. 
Copaescu et al.: A Classic Clinical Case: Neutrophilic Eccrine Hidradenitis

Generally, NEH is a self-limiting condition. Associations other than malignancy or chemotherapy have been made in the literature. The treatment is symptomatic and mainly involves topical or systemic corticosteroids $[1,7,9]$. Nonetheless, corticosteroids should be used with caution in neutropenic patients. Pain should be managed with usual analgesic medication if tender lesions are present. One study in a healthy patient reports colchicine use with improvement after 1 month [10]. As for the prevention of possible recurrences of NEH with the same drug use, one case report encourages dapsone use, which has specific effects on neutrophil migration, with a dosage of $100 \mathrm{mg}$ daily for $48 \mathrm{~h}$ before the treatment [11].

\section{Conclusion}

We have reported a febrile neutropenic patient undergoing chemotherapy for acute myelogenous leukemia who presented classic NEH lesions. The differential diagnosis includes cellulitis, and thus we emphasize the necessity of a prompt diagnosis in order to prevent the use of multiple antibiotics. In our case, cytarabine was the drug likely responsible for NEH.

\section{Disclosure Statement}

The authors declare no conflicts of interest.

\section{References}

1 Wallach D: Les dermatoses neutrophiliques. Rev Med Interne 2005;26:41-53.

-2 Aractingi S, et al: Neutrophilic dermatoses during granulocytopenia. Arch Dermatol 1995;131:1141-1145.

3 Solimando DA, Waddell JA: Guide to Combination Cancer Chemotherapy Regimens. St. Louis, Thomas Land Publishers, 2013, pp 342-344.

4 Bachmeyer C, Aractingi S: Neutrophilic eccrine hidradenitis. Clin Dermatol 2000;18:319-330.

5 Srivastava M, et al: Neutrophilic eccrine hidradenitis masquerading as facial cellulitis. J Am Acad Dermatol 2007;65:693-696.

-6 Bardenstein D, et al: Neutrophilic eccrine hidradenitis simulating orbital cellulitis. Arch Ophthalmol 1994;112:1460-1463.

7 Keane F, et al: Neutrophilic eccrine hidradenitis in two neutropaenic patients. Clin Exp Dermatol 2001;26:162-165.

-8 Thorisdottir KT, Kenneth J, Bergfeld WF, Andresen SW: Neutrophilic eccrine hidradenitis. J Am Acad Dermatol 1993;28:775-776.

-9 Sayed FE, et al: Neutrophilic eccrine hidradenitis to acetaminophen. J Eur Acad Dermatol Venereol 2006;20:1338-1340.

10 Belot V, et al: Adult idiopathic neutrophilic eccrine hidradenitis treated with colchicine (in French). Presse Med 2006;35:1475-1478.

11 Shear N, et al: Dapsone in the prevention of recurrent neutrophilic eccrine hidradenitis. J Am Acad Dermatol 1996;35:819-822.

Rouanet I, et al: Neutrophilic eccrine hidradenitis in an HIV-1-infected patient. AIDS 2012;26:775-776.
Bachmeyer C, Reygagne P, Aractingi S: Recurrent neutrophilic eccrine hidradenitis in an HIV-1-infected patient. Dermatology 2000;200:328-330.

14 Krische J, et al: Neutrophil eccrine hidradenitis in a patient with AIDS. J Dermatol 1998;25:199-200.

15 Oono T, et al: A case of infectious eccrine hidradenitis. J Dermatol 2006;33:142-145.

16 Takai T, Matsunaga A: A case of neutrophilic eccrine hidradenitis associated with streptococcal infectious endocarditis. Dermatology 2006;212:203-205.

17 Combemale P, et al: Neutrophilic eccrine hidradenitis secondary to infection with Serratia marcescens. $\mathrm{Br}$ J Dermatol 2000;142:784-788.

18 Antonovich D, et al: Infectious eccrine hidradenitis caused by Nocardia. J Am Acad Dermatol 2004;50:315318. 
Copaescu et al.: A Classic Clinical Case: Neutrophilic Eccrine Hidradenitis

Table 1. Chemotherapy protocol

\begin{tabular}{lll}
\hline Chemotherapy & Dose & Days \\
\hline Cytarabine & $175 \mathrm{mg}$ IV in $250 \mathrm{ml} \mathrm{NS} ; 12 \mathrm{ml} / \mathrm{h}$; continuous & $1-7$ \\
Idarubicin & $21 \mathrm{mg} \mathrm{IV} \mathrm{in} 50 \mathrm{ml} \mathrm{NS}$; $15 \mathrm{~min}$ DIE & $1-3$ \\
\hline
\end{tabular}

NS = Normal saline; DIE $=$ once a day.

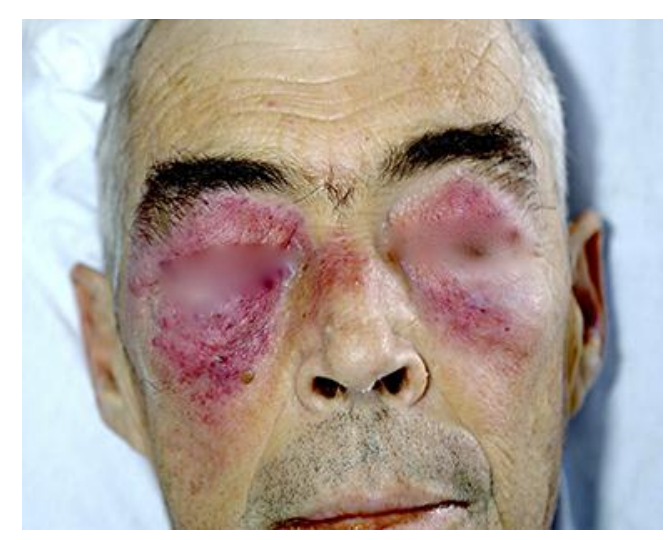

Fig. 1. Violaceous and oedematous plaques on the right and left periorbital areas and on the nose root at day 12 of induction therapy with cytarabine and idarubicin.

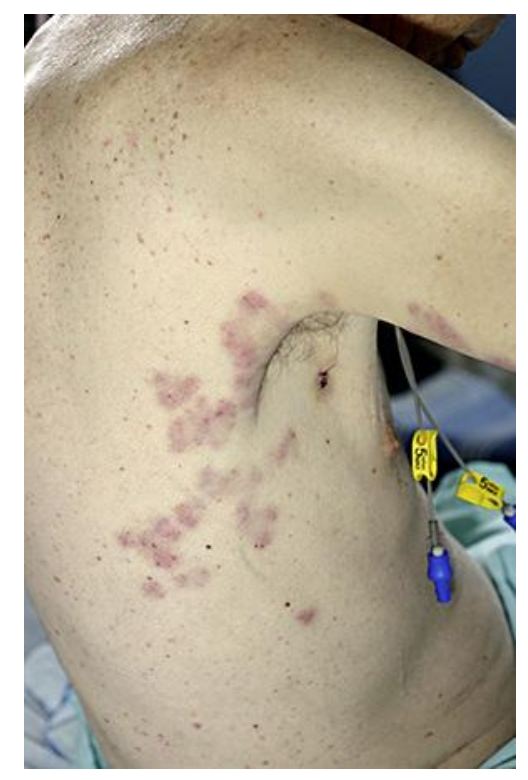

Fig. 2. Dry, non-scaly, round and well-delimited violaceous plaques on the right upper back. The site of the biopsy is shown. 


\section{Case Reports in Dermatology}

\begin{tabular}{l|l}
\hline Case Rep Dermatol 2013;5:340-346 \\
\hline DOI: 10.1159/000356229 & $\begin{array}{l}\text { C 2013 S. Karger AG, Basel } \\
\text { www.karger.com/cde }\end{array}$ \\
\hline
\end{tabular}

Copaescu et al.: A Classic Clinical Case: Neutrophilic Eccrine Hidradenitis

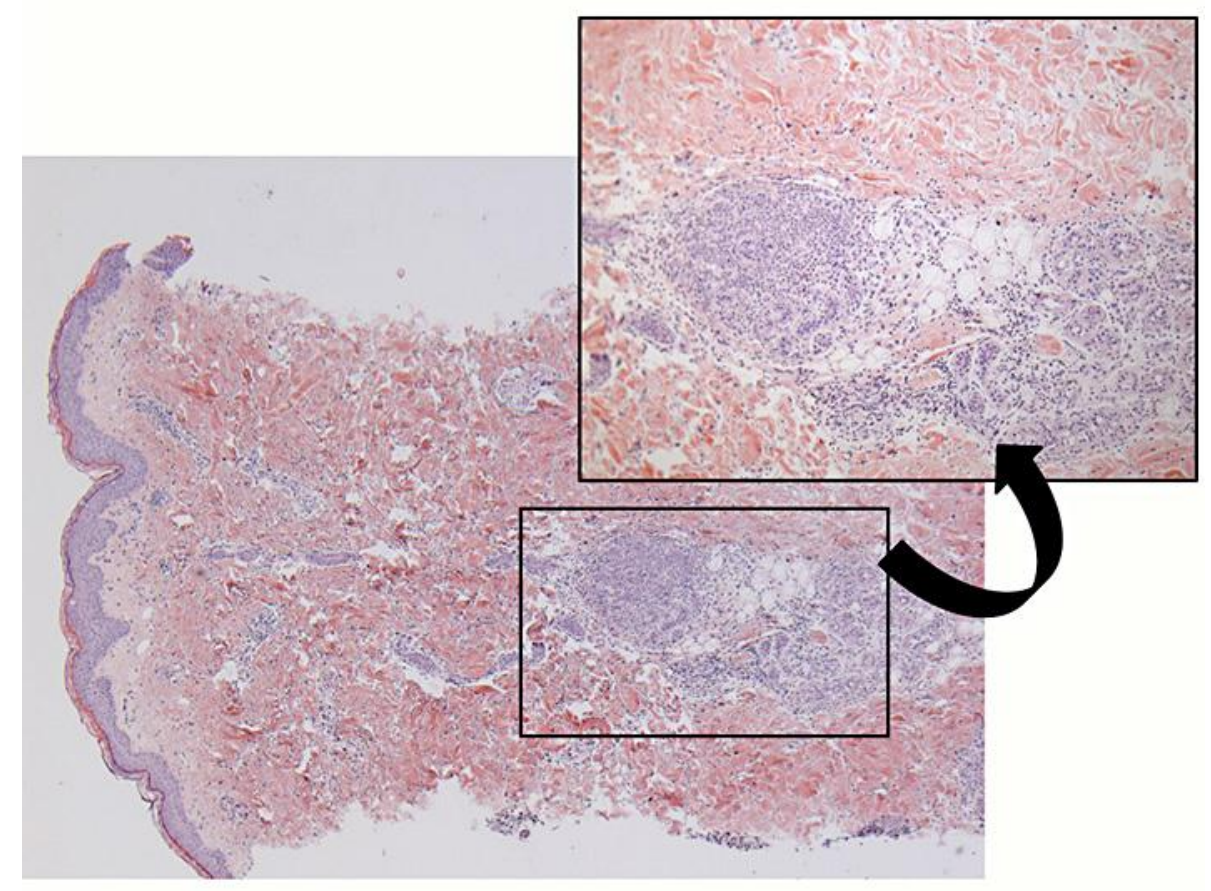

Fig. 3. Low and medium power view. Inflammation surrounding the eccrine secretory coils.

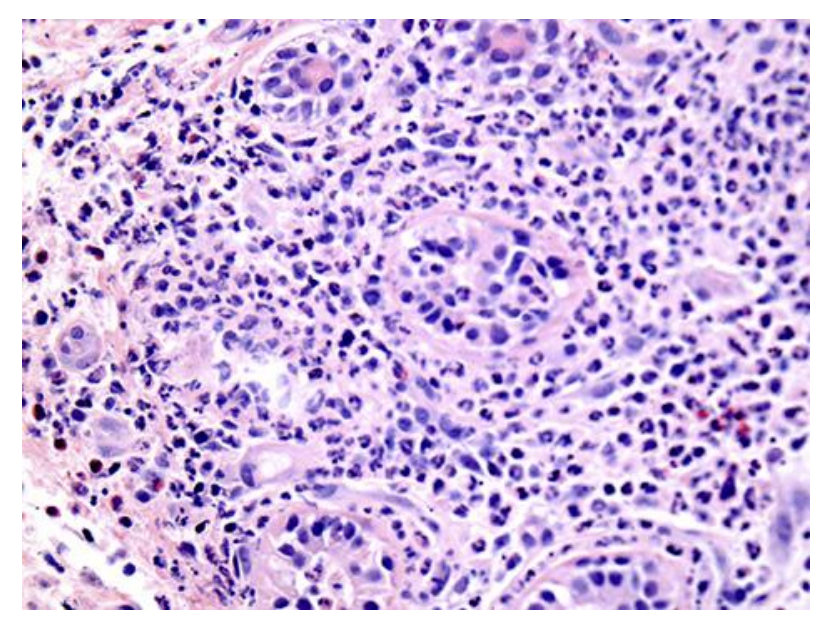

Fig. 4. Close-up view. Neutrophils surrounding and infiltrating the sweat gland epithelium. 